\title{
Chestnut's Obstetric Anesthesia Principles and Practice, Fifth Edition
}

\author{
David H. Chestnut, Cynthia A. Wong, Lawrence C. Tsen, Warwick D. Ngan Kee, Yaakov \\ Beilin, Jill M. Mhyre. Philadelphia: Elsevier Saunders, 2014, 1328 pages, \$324.00. ISBN: \\ 978-1-4557-4866-2
}

\author{
Allana Munro, MD • Ronald B. George, MD
}

Received: 25 February 2015/Accepted: 9 April 2015/Published online: 17 April 2015

(C) Canadian Anesthesiologists' Society 2015

It has been five years since the last edition of Obstetric Anesthesia Principles and Practice was released. In the interim, there has been significant change in obstetric anesthesia practice, with an emphasis on ultrasound for regional anesthesia, noninvasive technologies for hemodynamic monitoring, and new labour analgesia modalities for delivery. These innovations are successfully incorporated into the fifth edition of the textbook which consists of 55 chapters descriptively organized into ten parts.

George Santayana once said, "Those who cannot remember the past are condemned to repeat it". As in previous editions, this text begins with a thorough review of the history of obstetrical anesthesia. The text then moves on to maternal and fetal physiology, an essential chapter outlining the physiologic changes of pregnancy; updated figures show the effects of pregnancy on central hemodynamics and respiratory mechanics. A new section on sleep disorders is a timely addition, as the adverse physiologic effects of these conditions on the parturient are now being evaluated.

Fetal and neonatal assessment and therapy are addressed in five updated chapters. The chapter on intrapartum fetal assessment and therapy comprises a review of the threetiered fetal heart rate interpretation system. This is followed by the American Congress of Obstetricians and Gynecologists (ACOG) 2010 table of intrauterine resuscitative measures for fetal heart rate tracings, including measures to promote fetal oxygenation, improve uteroplacental blood flow, reduce uterine

A. Munro, MD · R. B. George, MD ( $ه)$

IWK Health Centre, Dalhousie University, Halifax, NS, Canada

e-mail: rbgeorge@dal.ca activity, and alleviate umbilical cord compression. The American Heart Association 2011 algorithm for neonatal resuscitation reflects current changes, including the omission of naloxone use for primary resuscitation. Fetal and neonatal neurologic injury enjoys a new chapter with a discussion of fetal brain development and a full description of neurogenesis and neurotransmitter systems. New information is provided from the ACOG 2010 opinion regarding antenatal maternal magnesium for neuroprotection. The section on anesthesia and brain injury has been expanded considerably and now includes the controversial topic of early childhood anesthetic exposure and functional impairment.

The portion of the textbook dedicated to anesthesia for labour and vaginal delivery begins with a discussion on the obstetric management of labour and delivery and includes the recent positions of the ACOG and the American Academy of Family Physicians on a trial of labour and vaginal birth after Cesarean delivery. The pain of childbirth is thoroughly reviewed, including chapters that address the non-pharmacologic, systemic, and neuraxial analgesia options for labour. In keeping with modern practice, the use of ultrasound for neuraxial anesthesia is highlighted. This skill has become increasingly important as the obstetric population ages and develops additional comorbidities, including obesity. This topic is followed by a discussion on anesthesia for Cesarean delivery. A new author delivers a detailed discussion on the mechanism and prevalence of pain in the chapter on postoperative and chronic pain following childbirth. This part of the textbook provides the anesthesiologist with systemic and regional options for postoperative analgesia.

The difficult airway, always an important chapter, continues to be highlighted. The authors have included 
tables of case fatality rates, a revised section on rapid sequence induction and cricoid pressure, and a full discussion on awake intubation before general anesthesia. This description provides the reader with a complete reference about a topic with considerable potential for morbidity and mortality in obstetrical anesthesia.

The section on obstetrical hemorrhage has been modified considerably and contains new additions outlining a protocol-driven and team-based response to hemorrhage for the prevention of mortality. Authors have included a table on transfusion trigger thresholds from the 2012 modified early obstetric warning system. The difficult decision regarding the administration of blood products is tackled by a discussion of the risks and an exploration of blood conservation techniques and concludes with the treatment of massive blood loss.

In the section on the parturient with systemic disease, Chestnut has once again successfully captured the important obstetrical medical comorbidities. The information on cardiovascular disease has been reorganized and expanded with new emphasis on cardiac imaging to complement the current standards of medical practice. A welcome addition to the text is a new chapter on psychiatric disorders which provides a brief overview of the most common psychiatric comorbidities seen in the obstetric population. Most importantly, there is information on the management of these diseases and their associated treatments in pregnancy.
Critical care is a significant addition to this release. Initial assessment and resuscitation is emphasized early in the chapter to reflect its importance. This is followed by the Advanced Trauma Life Support approach with primary and secondary surveys, labs, and imaging, as well as the 2008 algorithm for managing the pregnant woman after trauma. The 2010 American Heart Association guidelines for Cardiopulmonary Resuscitation, management of a brain dead patient, and common themes in critical care medicine are covered before the chapter concludes with the effects on fetal outcome during maternal critical illness.

The text as a whole has been edited to reflect current anesthesia practice. As in previous editions, the chapters remain an easy enjoyable read with solid evidence-based recommendations. The outline of the textbook remains similar to previous editions, contributing to the readability. The information boxes combined with key points following each chapter allow the reader to refer to crucial information at a glance. While still lacking colour, the figures, tables, and boxes have been updated and merge well with the surrounding text. The 55 chapters and four appendices have been considerably updated since the last edition. Chestnut and his 79 expert contributors have once again released a current guide to obstetric anesthesia that proves useful to both junior learners and subspecialty providers.

Conflicts of interest None declared. 